\title{
Increased diffusivity in acute multiple sclerosis lesions predicts risk of black hole
}

R.T. Naismith, MD*

J. Xu, PhD*

N.T. Tutlam, MPH

P.T. Scully

K. Trinkaus, $\mathrm{PhD}$

A.Z. Snyder, PhD, MD

S.-K. Song, $\mathrm{PhD} \ddagger$

A.H. Cross, MD $\ddagger$

Address correspondence and reprint requests to Dr. Robert T. Naismith, Neurology,

Washington University, Campus Box 8111, 660 S. Euclid Ave., St. Louis, MO 63110

naismithr@neuro.wustl.edu

See also page 1702

Supplemental data at www.neurology.org

\section{ABSTRACT}

Objective: Diffusion tensor imaging (DTI) quantifies Brownian motion of water within tissue. Inflammation leads to tissue injury, resulting in increased diffusivity and decreased directionality. We hypothesize that DTI can quantify the damage within acute multiple sclerosis (MS) white matter lesions to predict gadolinium (Gd)-enhancing lesions that will persist 12 months later as T1 hypointensities.

Methods: A cohort of 22 individuals underwent 7 brain MRI scans over 15 months. DTI parameters were temporally quantified within regions of $\mathrm{Gd}$ enhancement. Comparison to the homologous region in the hemisphere contralateral to the $\mathrm{Gd}$-enhancing lesion was also performed to standardize individual lesion DTI parameters.

Results: After classifying each Gd-enhancing region as to black hole outcome, radial diffusivity, mean diffusivity, and fractional anisotropy, along with their standardized values, were significantly altered for persistent black holes ( $\mathrm{PBH}$ ), and remained elevated throughout the study. A Gd-enhancing region with a $40 \%$ elevation in radial diffusivity had a 5.4-fold (95\% confidence interval [CI]: 2.1,13.8) increased risk of becoming a $\mathrm{PBH}$, with $70 \%(95 \% \mathrm{Cl}: 51 \%, 85 \%)$ sensitivity and 69\% (95\% Cl: 57\%, 80\%) specificity. A model of radial diffusivity, with volume and length of $\mathrm{Gd}$ enhancement, was associated with a risk of becoming a $\mathrm{PBH}$ of 5.0 (95\% Cl: 2.6 , 9.9). Altered DTI parameters displayed a dose relationship to duration of black hole persistence.

Conclusions: Elevated radial diffusivity during gadolinium enhancement was associated with increased risk for development of a persistent black hole, a surrogate of severe demyelination and axonal injury. An elevated radial diffusivity within active multiple sclerosis lesions may be indicative of more severe tissue injury. Neurology ${ }^{\circledR}$ 2010;74:1694-1701

\section{GLOSSARY}

ABH = acute black hole; $\mathbf{C B H}=$ chronic black hole; DTI = diffusion tensor imaging; FLAIR = fluid-attenuated inversion recovery; $\mathbf{G d}=$ gadolinium; $\mathbf{M S}=$ multiple sclerosis; $\mathbf{N A W M}=$ normal-appearing white matter; $\mathbf{P B H}=$ persistent black hole; $\mathbf{R D}=$ radial diffusivity; $\mathbf{R O I}=$ region of interest; $\mathbf{T} 1 \mathbf{H}=$ chronic $\mathrm{T} 1$ hypointensity; $\mathbf{T B H}=$ transient black hole .

In multiple sclerosis (MS), inflammation leads to variable myelin and axon injury. ${ }^{1}$ Tissue destruction can increase the risk for worse relapse recovery, permanent disability, and future disease progression..$^{2-4}$ Diffusion tensor imaging (DTI) may serve as a quantitative measure of tissue damage and provide information regarding the pathologic substrate.

Gd-enhancing lesions represent increased permeability of the blood-brain barrier with infiltration of inflammatory cells. Some Gd-enhancing lesions resolve with little consequence to the tissue, while others progress to extensive and permanent parenchymal loss. ${ }^{5}$ Chronic T1 hy-

\footnotetext{
*The two first authors contributed equally on this project and manuscript.

¥The two senior authors contributed equally on this project and manuscript.

From the Departments of Neurology (R.T.N., J.X., N.T.T., A.Z.S., A.H.C.), Radiology (P.T.S., A.Z.S., S.-K.S.), and Biostatistics (K.T.), Washington University, St. Louis, MO.

Study funding: Supported by National Institutes of Health (K23NS052430-01A1 to R.T.N., K12RR02324902 to R.T.N., K24 RR017100 to A.H.C.); the National MS Society (FG1782A1 to J.X., CA1012 to A.H.C. and S.K.S., RG 3670 to S.K.S.); and the Manny and Rosalyn RosenthalDr. John L. Trotter Chair in Neuroimmunology from Barnes-Jewish Hospital Foundation (A.H.C.). This publication was made possible by grant number UL1 RR024992 from the National Center for Research Resources (NCRR), a component of the NIH and NIH Roadmap for Medical Research. This research was also supported in part by NIH grants CO6 RR020092 and RR024992 (Washington University Institute of Clinical and Translational Sciences-Brain, Behavioral and Performance Unit). Its contents are solely the responsibility of the authors and do not necessarily represent the official view of NCRR or NIH.

Disclosure: Author disclosures are provided at the end of the article.
} 
pointensities (T1Hs), or chronic black holes (CBHs), have direct histopathologic correlation to severe tissue damage, based on degree of axon loss and demyelination at autopsy. ${ }^{6,7}$ Acute black holes (ABHs) are T1Hs with Gd enhancement, observed on the concurrent noncontrast T1-weighted scans. ${ }^{8,9}$ ABHs represent a heterogeneous group of lesions, with variable contributions from edema, demyelination, and axonal injury. A minority of $\mathrm{ABH}$ s will become persistent black holes (PBHs) after 12 months of imaging follow-up; those that recover to become isointense are designated as transient black holes (TBHs).

The present study sought to determine whether DTI at lesion onset would predict longterm tissue damage associated with PBHs. In animal studies of acute inflammatory lesions within white matter tracts, directional (axial and radial) diffusivities may serve as surrogates of axon and myelin pathologies. ${ }^{10-13}$ Hypotheses for the present study were that a drop in axial diffusivity preceding Gd enhancement, and a rise in radial diffusivity during or after Gd enhancement, would predict development of a $\mathrm{PBH}$.

METHODS Standard protocol approvals and patient consent. All subjects provided informed consent, after approval by the Washington University Human Research Protection Office/Institutional Review Board.

Study protocol. Seven brain MRIs were obtained over the course of 15 months as part of a cohort study of rituximab add-on therapy for breakthrough disease in MS. Included were those with a relapse within the past 12 months and at least 1 enhancing lesion on 1 of 3 monthly pretreatment MRIs. Subjects continued on their injectable therapy and received rituximab after their third baseline/pretreatment MRI. Rituximab was dosed at $375 \mathrm{mg} / \mathrm{m}^{2}$ IV for 4 weekly doses. MRI was obtained at months $-2,-1,0,3,4$, and 5 to determine whether rituximab would decrease new enhancing lesions. A follow-up MRI was obtained at least 12 months from the resolution of the last enhancing lesion to determine whether a subsequent $\mathrm{PBH}$ developed.

MRI protocol. Patients were imaged on a 1.5 T MRI scanner (Sonata, Siemens Medical Solutions, Germany) using an 8-channel phased-array head coil. Axial DTI data were acquired in 14 minutes using a single-shot spin-echo echoplanar imaging sequence with diffusion encoding in 6 noncollinear directions, 6 averages, and a resolution of $2.5 \times 2.5 \times 2.0 \mathrm{~mm}^{3}$. Regions of interest (ROIs) delineated on anatomic images were in register with the DTI data, and ROIs defined on any anatomic image on any scan session were transferrable to the DTI data on any scan session. Details on the MRI sequences and coregistration methods can be found in e-Methods on the Neurology ${ }^{\circledR}$ Web site at www.neurology.org.

Region of interest analysis. Amira v4.0 (Visage Imaging) was used for manual segmentation, DTI quantification, and classification of T1 hypointensities. ROIs were drawn on the T1W postcontrast image, blinded to the black hole outcome measure. When there was an acute black hole with Gd ring enhancement, the entire region was segmented including the nonenhancing acute $\mathrm{T} 1 \mathrm{H}$ center, if a previous MRI confirmed no lesion prior to the acute black hole. If a region of enhancement was adjacent or peripheral to a preexisting black hole, as defined by an MRI previous to enhancement, then that preexisting black hole was not included within the ROI. Gd-enhancing lesions were excluded if excessively small $\left(<16 \mathrm{~mm}^{3}\right)$.

The mean Gd-region DTI in this population was examined over time. To evaluate changes within individual Gd-enhancing lesions, a DTI ratio standardized to an internal control value was obtained by selecting the same region in the contralateral hemisphere, based upon distance from midline on the $b_{0}$ image. The FA map was used to ensure that the contralateral control ROI was in a region homologous to the lesion ROI. If the contralateral ROI was not in the same homologous tract as the lesion, it was shifted 1-3 mm. Adjustments were made blinded to the outcome. To avoid partial volume effect, the FA map was also used to exclude voxels bordering CSF or cortex.

The goal of selecting the contralateral control region was to include an area of white matter that was normal as assessed on the fluid-attenuated inversion recovery (FLAIR) image (NAWM). Due to the presence of contralateral T2 hyperintensities $(\mathrm{T} 2 \mathrm{H})$, obtaining a corresponding NAWM ROI was not possible in $35 \%$ of lesions. In order to preserve the power of the study by not excluding lesions in which contralateral NAWM was unobtainable, the control lesion ROI was categorized as to whether it was pure NAWM or contained some T2H. These two categorizations were then utilized as a covariate in the statistical modeling to adjust for any confounding. The T1-hypointensity was determined by visual inspection, compared to surrounding normal appearing tissue.

Statistics. Mean DTI values were determined by a linear mixed model to account for each subject contributing a variable number of lesions, along with repeated measures over time. For modeling, DTI parameters were transformed into a $\log$ scale for axial diffusivity and radial diffusivity (RD), a square root scale for FA, and an inverse scale for MD in order to form Gaussian distributions. Classification of the control region as NAWM or T2H revealed an interaction with all DTI parameters ( $p<0.01)$, justifying the use of this covariate within the linear mixed model. The clinical covariates of age, disease duration, and Expanded Disability Status Score were assessed in the model, did not contribute significantly, and were not included. Clustered logistic regression was used to determine risk of progression to a $\mathrm{PBH}$, with covariates of lesion volume and months of Gd enhancement.

RESULTS Demographics. Subject demographics are presented in table 1 . The 22 subjects had MS for a median of 9.1 years with median Expanded Disability Status Scale score of 4.4 .

Longitudinal control ROI remained stable. Each Gdenhancing lesion ROI had a homologous ROI located in the contralateral hemisphere. DTI parameters of the control ROIs in the contralateral 
Table $1 \quad$ Baseline subject demographics

\begin{tabular}{|c|c|}
\hline Characteristics & Values \\
\hline No. & 22 \\
\hline Age, $y$, median (range) & $42.0(33-53)$ \\
\hline Gender & 15 women, 7 men \\
\hline Disease duration, $y$, median (range) & $9.1(2-23)$ \\
\hline EDSS, median (range) & $4.4(2-6.5)$ \\
\hline IFN $\beta$-1b SC, n & 6 \\
\hline IFN $\beta-1 \mathrm{a}$ IM, n & 6 \\
\hline IFN $\beta$-1a SC, n & 7 \\
\hline Glatiramer acetate, $n$ & 3 \\
\hline $\begin{array}{l}\text { T2 lesion burden, } \mathrm{mm}^{3} \text {, } \\
\text { median (range) }\end{array}$ & 20.5 (5.3-53.5) \\
\hline $\begin{array}{l}\text { T1 lesion burden, } \mathrm{mm}^{3} \text {, } \\
\text { median (range) }\end{array}$ & $1.9(0-25.1)$ \\
\hline $\begin{array}{l}\text { Gadolinium lesions, mm }{ }^{3} \text {, } \\
\text { median (range) }\end{array}$ & $0.58(0.1-10.2)$ \\
\hline
\end{tabular}

Abbreviations: EDSS $=$ Expanded Disability Status Scale; IFN $\beta=$ interferon- $\beta$.

hemisphere did not change over 15 months (figure e-1). This affirmed the feasibility of using the contralateral hemisphere to standardize individual Gdenhancing lesion DTI measurements within brain regions by the ratio of the lesion ROI vs the contralateral ROI.

DTI parameters became altered during Gd enhancement. Ninety-five Gd-enhancing lesions were identified (table 2). For all 95 Gd-enhancing lesions, RD increased by $38 \%$, FA decreased by $35 \%$, and MD increased by $21 \%$ compared to the corresponding contralateral ROI (figure 1). Axial diffusivity was not appreciably changed when evaluating all lesions regardless of black hole outcome. Fifty-six percent of lesions had at least 1 MRI prior to Gd enhancement.

\begin{tabular}{|ll|}
\hline Table 2 Lesion characteristics & \\
\hline Characteristics & Values (\%) \\
\hline Total GdE lesions & 95 \\
\hline ABH & $49(53)$ \\
\hline ABH resolved with Gd resolution & $13(14)$ \\
\hline TBH resolved 1-3 mo & $6(6)$ \\
\hline TBH resolved $>$ 3 and <12 mo & $7(7)$ \\
\hline No black holes during Gd & $46(48)$ \\
\hline PBH & $28(30)^{\mathrm{a}}$ \\
\hline$\%$ Enhancement $>1$ mo & $41(43)$ \\
\hline$\%$ Re-enhancement & $5(5)$ \\
\hline
\end{tabular}

Abbreviations: $\mathrm{ABH}=$ acute black hole; $\mathrm{GdE}=$ gadolinium enhancing; $\mathrm{PBH}=$ persistent black hole; $\mathrm{TBH}=$ transient black hole.

a Five $\mathrm{PBH}$ s arose from lesions that were not $\mathrm{ABH}$ s during Gd enhancement, but became T1-hypointense the month after $\mathrm{Gd}$ resolution.
At -1 and -2 months prior to enhancement, there was a nonsignificant trend for progressive alteration of $\mathrm{RD}, \mathrm{FA}$, and $\mathrm{MD}$ within the future lesion ROI. Within 2 months after enhancement, these 3 parameters began to normalize toward preenhancement baseline (figure 1).

DTI parameters predicted enhancing lesions that became PBHs. RD, MD, and FA distinguished enhancing lesions that would become $\mathrm{PBHs}$ (table 3). Compared to the -1 month prior to Gd enhancement, mean and ratio of $\mathrm{RD}$ for lesions destined as $\mathrm{PBH}$ s were significantly elevated at the time of enhancement (figure 2). After at least 12 months, RD, both mean and standardized ratio, continued to separate the $\mathrm{PBH}$ s from isointense regions. $\mathrm{RD}$ in $\mathrm{PBH} s$ remained elevated at 12 months compared to pre-Gd enhancement baseline. Similarly, higher MD and lower FA at time of Gd enhancement each predicted black hole persistence at 12 months.

For lesions that enhanced for over 1 month, time 0 in the analyses above was based upon the month of maximal Gd enhancement. Additional analyses determined whether the discriminatory ability of DTI upon Gd enhancement would remain valid if monthly MRIs were not being obtained. If a random month when the lesion was Gd enhancing was set as time $0, \mathrm{PBH}$ continued to be predicted using $\mathrm{RD}$ and $\mathrm{MD}$ ( $p<0.05$, modeling with 500 repetitions).

Only one subject displayed enhancing lesions $(n=10)$ after rituximab therapy. In the event that the timing of rituximab therapy affected the outcome of $\mathrm{ABH}$ s, the analyses were also performed excluding that subject. PBHs were still predicted at the time of enhancement using the parameters of radial diffusivity ratio $(p<0.01)$, MD ratio $(p=0.001)$, and FA ratio $(p<0.01)$.

DTI parameters demonstrated a relationship to time of black hole persistence. To determine if a relationship existed between degree of diffusivity abnormalities and the length of time the black hole persisted, each individual diffusion parameter measured at month 0 was assessed for a linear change over time. Radial diffusivity (ANOVA $p<0.001)$, FA $(p=0.001)$, and MD $(p=0.001)$ ratios demonstrated a significant linear trend for increasing diffusivity and decreasing anisotropy with increasing time of black hole persistence (table e-1).

Radial diffusivity predicts individual lesions at risk for progression. Within this cohort, a Gd-enhancing lesion with a radial diffusivity ratio $\geq 1.4$ had an OR of 5.4 (2.1-13.8) for becoming a PBH when each lesion was analyzed as an independent event. A radial diffusivity ratio of $\geq 1.4$ predicted progression to a $\mathrm{PBH}$ at 12 months with a sensitivity of 70\% (51\%- 
Figure 1 Diffusion parameters over time in relation to gadolinium enhancement, all lesions included

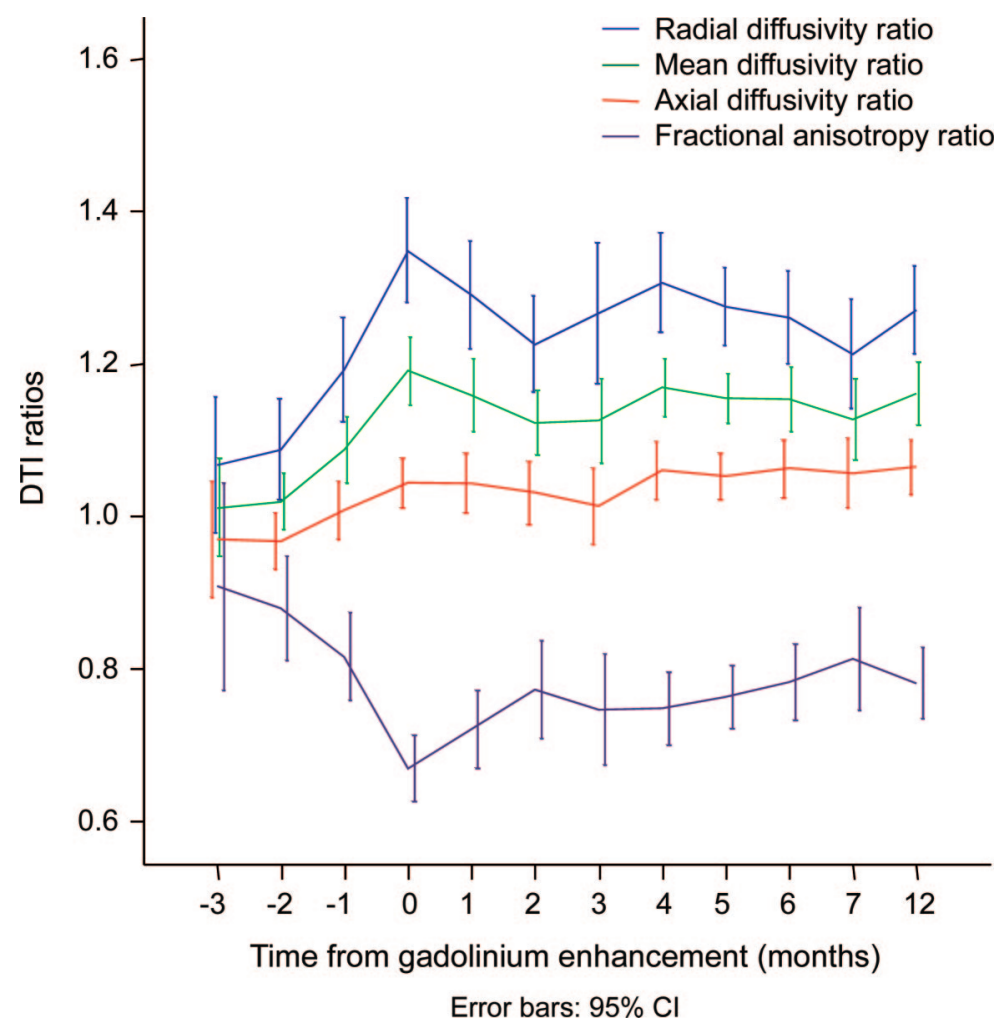

Radial diffusivity, mean diffusivity, and fractional anisotropy become altered at time of maximum gadolinium enhancement (month 0 ). These diffusion tensor imaging parameters return toward their month -1 baseline within 2 months (overlapping confidence intervals).

$85 \%)$ and specificity of $69 \%(57 \%-80 \%)$ for an individual lesion. When each lesion was evaluated by a clustered logistic model, controlling for the presence of $\mathrm{T} 2 \mathrm{H}$ on the contralateral side and repeated measures within subjects, the increased odds of becoming a $\mathrm{PBH}$ at 12 months for radial ratio $\geq 1.4$ remained 5.4 (standard error of 0.36 for the regression parameter). Additional covariates of lesion volume and Gd enhancement for greater than 1 month were evaluated, as both previously had been reported predictors of conversion to a $\mathrm{PBH} .{ }^{8}$ Radial ratio diffusivity with the 2 additional covariates demonstrated that an individual lesion with a radial diffusivity ratio $\geq 1.4$ had an OR of 5.0 (2.6-9.9) for becoming a PBH $(p<0.0001$, generalized estimating equations model).

Drop in axial diffusivity did not predict progression to PBH. No significant decrease in axial diffusivity prior to or at the time of enhancement for the $\mathrm{PBH}$ group was observed (figure 2), and no linear trend for increasing risk of $\mathrm{PBH}$ with decreasing axial diffusivity was found (ANOVA, $p=$ NS).

DISCUSSION We tested the predictive power of DTI at Gd enhancement for development of a PBH, a surrogate marker of severe tissue injury. $\mathrm{RD}$ was found to be a reliable and consistent parameter at the time of enhancement to distinguish outcome (table 3). A $40 \%$ elevation of $\mathrm{RD}$ within an individual enhancing lesion, in comparison to the homologous region within the opposite hemisphere, was associated with a 5.0-fold risk for black hole persistence at 12 months $(p<$ 0.0001). Likewise, a $40 \%$ elevation in RD predicted persistence as a black hole with a sensitivity and specificity of $70 \%$. Furthermore, the degree of increase in $\mathrm{RD}$ at Gd enhancement onset was correlated with the length of time the black hole was observed. Thus, increased $\mathrm{RD}$, as it relates to a chronic black hole, may suggest intense demyelination, axonal dropout, and overall loss of tissue integrity. ${ }^{14}$

The significance of $\mathrm{CBHs}$ has been established by autopsy and biopsy, wherein decreased axonal density, myelin loss, and matrix destruction have been noted in comparison to $\mathrm{T} 1$ isointense areas. ${ }^{6,15,16} \mathrm{~A}$ dose relationship between decreasing axonal counts (by silver staining) and increasing degree of $\mathrm{T} 1 \mathrm{hy}-$ pointensity has been shown. ${ }^{6}$ In addition, quantitative imaging modalities have confirmed notable abnormalities within black holes on a cross-sectional basis. Magnetic resonance spectroscopy has demonstrated a decreased NAA level, suggestive of axonal injury/loss. ${ }^{17,18}$ Quantitative magnetization transfer imaging has shown lower values for black holes, consistent with demyelination. ${ }^{19,20}$ Diffusion imaging studies have found an increase in water diffusion, consistent with tissue breakdown, in black holes. ${ }^{21-29}$ A relationship between the burden of chronic black holes and increasing disability has been established, supporting its clinical relevance..$^{30-33}$

DTI may provide complementary and additional information to other MRI techniques in clinical practice. Whereas T1 hypointensities and T2 hyperintensities are indicative of a threshold of increased water content, DTI can assess structural hindrances to the Brownian motion of water, providing information more specific for tissue breakdown. DTI can be implemented with current commercial software packages and hardware. Whole brain coverage with DTI can be achieved at a relatively high resolution within a practical time.

Population mean lesion DTI value may be useful for assessing a group of lesions over time, as in a clinical trial. In this study, the mean RD, FA, and MD for all lesions did distinguish PBH from transient black hole (table 3). However, DTI values vary greatly throughout the brain, and the mean value has limited practicality in lesions within individual patients. A solution is to standardize the DTI within each lesion by having each subject serve as their own control in a longitudinal assessment. The DTI ratio 


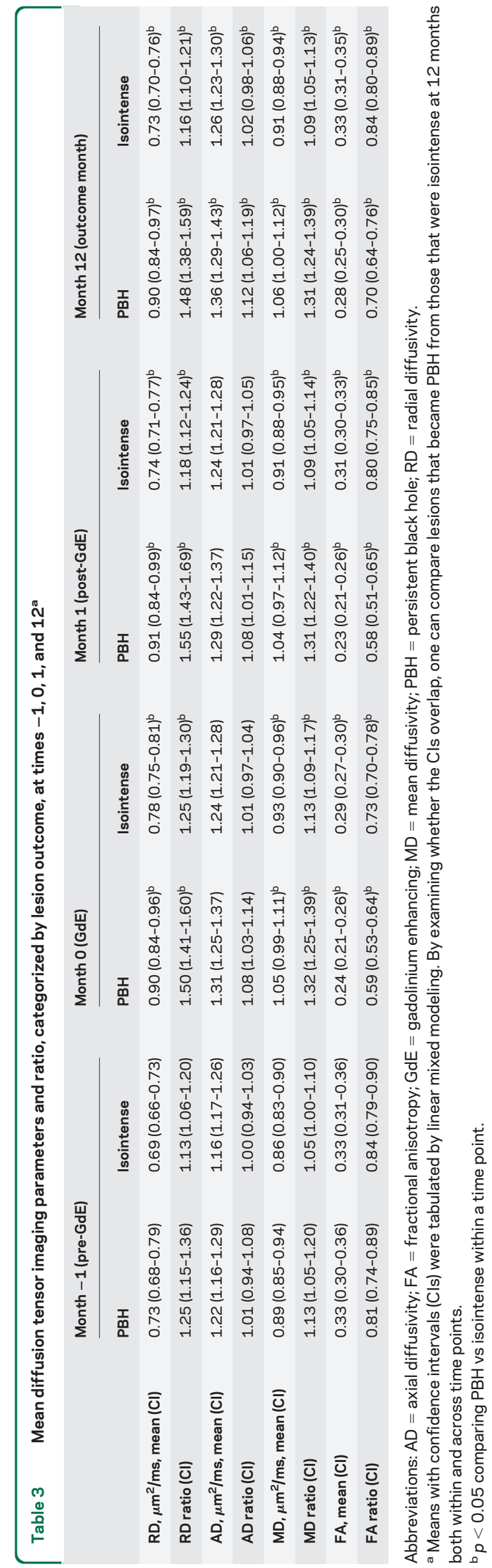

of the lesion and a homologous region in the opposite hemisphere has the advantage of not needing to rely upon a normal control common atlas space. One difficulty in using a comparative control atlas is that patients with MS have variable degrees of regionspecific atrophy. Although coregistering the same individual over time can be done reliably, attempting to warp the brain image of someone with MS onto a normal brain would be associated with misalignment errors. In the present study, regardless of whether the contralateral hemisphere was normal or contained $\mathrm{T} 2 \mathrm{H}$, either scenario found RD ratio to also be effective in predicting black holes.

As demonstrated in human optic neuritis, a drop in axial diffusivity may be among the first measurable DTI parameters to become altered within an acute lesion, before tissue breakdown results in overall increased diffusivity and decreased directionality. ${ }^{34,35}$ In the present study, axial diffusivity did not prove to be as useful as radial diffusivity, and axial diffusivity did not decrease. One explanation for the differences between the 2 studies is that the usefulness of axial diffusivity may be limited to tightly packed white matter tracts with relatively limited interstitial space. Subjects in this study had MS for median 9 years, whereas in the study of acute optic neuritis, subjects were assessed at demyelinating disease onset. Thus, in the present study some enhancing lesions may have occurred in regions of previous injury. A second reason is that changes in brain diffusivity may occur up to 4 months prior to enhancement, and the present study had insufficient power to examine DTI many months before $\mathrm{Gd}$ enhancement. ${ }^{36} \mathrm{~A}$ third reason is that, unlike the optic nerve, Gd-enhancing lesions were located in multiple regions of CNS white matter, including areas with low directionality and crossing fibers. In regions of low directionality, there may not be a robust principal eigenvector, and diffusivity can increase in many directions. ${ }^{37}$

$\mathrm{ABHs}$ that became isointense demonstrated normalization of diffusivity over time (table 3 , overlapping confidence intervals from month -1 to months 1 and 12), perhaps reflecting resolution of edema associated with the acute inflammatory infiltrate. Late normalization of diffusivity may be due to remyelination, continued resolution of inflammation, or both. However, only $7 \%$ of lesions fell into the late resolution category (hypointense until $>3$ and $<12$ months), resulting in the study being underpowered to test this hypothesis.

The limitations of this study include its modest sample size and the unique type of MS patient studied. The 22 subjects enrolled had breakthrough disease with active lesions, long disease durations, and higher Expanded Disability Status Scale scores than 
Figure 2 Radial diffusivity over time in relation to gadolinium (Gd) enhancement, categorized by black hole outcome

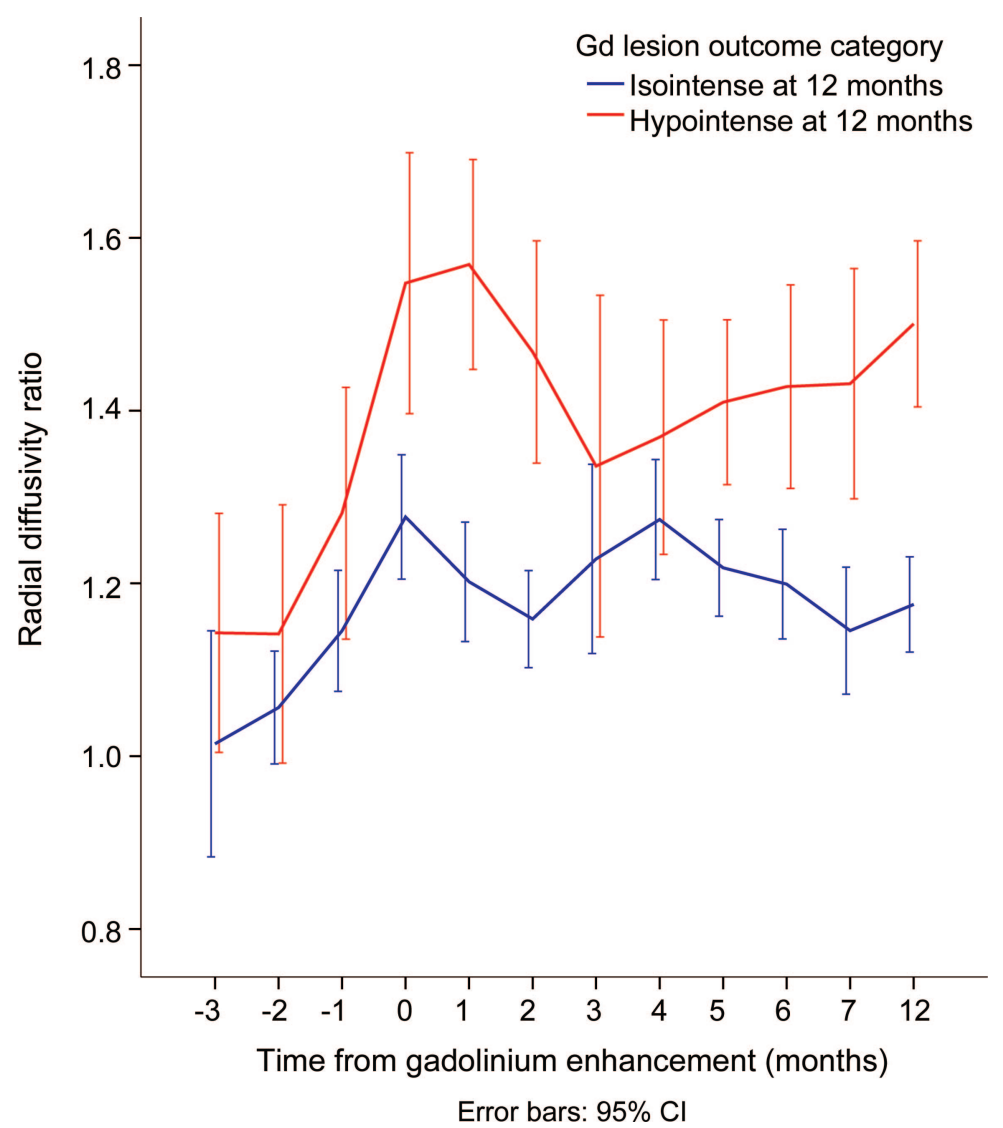

During maximum Gd enhancement (time 0), radial diffusivity becomes elevated over baseline (time -1), regardless of lesion outcome. However, for lesions destined to become persistent black holes, radial diffusivity is larger than for lesions that do not become black holes. In addition, it remains elevated throughout the duration of follow-up.

in many trials of relapsing MS. The incidence of developing $\mathrm{PBH}$ in this group may be higher than in patients with MS with early disease. Black holes are a qualitative imaging parameter, and are not equivalent to the gold standard of tissue histopathology. However, obtaining tissue in living patients is impractical, and evaluating the temporal evolution of acute inflammatory lesions blinded to the outcome of PBH can still help establish a connection between DTI and focal injury. Finally, re-enhancing lesions might alter the $\mathrm{T} 1 \mathrm{H}$ outcome if a second wave of destructive inflammation were to develop after measuring initial DTI. However, only 5 of the 95 lesions in this study were noted to be re-enhancing, representing a minority of lesions that would be unlikely to alter the result.

A future study should include the complete spectrum of MS subtypes, validation of the $40 \%$ increase in radial diffusivity in another population, and monthly DTI over a longer time period to better define tissue alterations prior to enhancement and after black hole resolution. Incorporating magnetiza- tion transfer and spectroscopy with DTI would define the role of each modality over time, and in relation to one another. ${ }^{38-40}$ Such studies should correlate DTI parameters with clinical disability outcomes, to determine if DTI can identify suboptimal responses to treatment prior to clinical identification.

DTI may have value in clinical practice to probe the structural integrity of new lesions that occur despite therapy, such as in this study. DTI of active lesions may be useful as a surrogate marker in clinical trials of anti-inflammatory and neuroprotective agents. Quantitative DTI may complement other imaging techniques to assist in the assessment of new therapeutics and in early determination of treatment response.

\section{AUTHOR CONTRIBUTIONS}

Statistical analysis was conducted by Dr. Trinkaus.

\section{DISCLOSURE}

Dr. Naismith has received travel expenses and/or honoraria for lectures and educational activities from Bayer Schering Pharma, Biogen Idec, Teva Pharmaceutical Industries Ltd., and Elan Corporation; serves on speakers' bureaus for Bayer Schering Pharma, Biogen Idec, Elan Corporation, and Teva Pharmaceutical Industries Ltd.; and receives research support from Acorda Therapeutics Inc., the NIH (K23NS052430-01A1 [PI] and K12RR02324902 [PI]), and the National MS Society. Dr. Xu, N.T. Tutlam, P.T. Scull, and Dr. Trinkaus report no disclosures. Dr. Snyder serves as Action Editor of the Journal of Computational Neuroscience and receives research support from the NIH (5 P50 NS006833 [Core B PI], NINDS P30 NS 048056 [Sub-I], NIMH R01 MH 071920 [Sub-I], P50 AG05681 [Sub-I], NIA P01 AG026276 [Sub-I], 1 R01 DK64832-05 [Sub-I], 5 R01 NS037237-08 [Sub-I], and 5 R01 MH074916 [Sub-I]). Dr. Song reports no disclosures. Dr. Cross serves on scientific advisory boards for Eli Lilly and Company, Genentech, Inc., and Biogen Idec; serves on the editorial boards of Brain Pathology and the Journal of Neuroimmunology and as an editor and contributor to CONTINUUM; receives royalties from the publication of Handbook of Multiple Sclerosis, 4th Ed. (Taylor \& Francis Group, 2006); serves on speakers' bureaus for Bayer Schering Pharma and Biogen Idec; has received speaker honoraria from Amgen and Pfizer Inc.; and receives research support from Sanofi-Aventis, Acorda Therapeutics Inc., Genentech Inc., Biogen Idec, the NIH (NINDS PO1 NS059560-01 [PI], NINDS UO1 NS45719-01A1 [CoI], RO1 NS047592 [Co-I], NINDS RO1 NS 051591 [PI]), ICTS Washington University, the National MS Society USA, Consortium of Multiple Sclerosis Centers, and the Barnes-Jewish Hospital Foundation.

Received August 21, 2009. Accepted in final form February 3, 2010.

\section{REFERENCES}

1. Trapp BD, Peterson J, Ransohoff RM, Rudick R, Mork S, Bo L. Axonal transection in the lesions of multiple sclerosis. N Engl J Med 1998;338:278-285.

2. Bjartmar C, Trapp BD. Axonal and neuronal degeneration in multiple sclerosis: mechanisms and functional consequences. Curr Opin Neurol 2001;14:271-278.

3. Trapp BD, Ransohoff R, Rudick R. Axonal pathology in multiple sclerosis: relationship to neurologic disability. Curr Opin Neurol 1999;12:295-302.

4. Lassmann H. Axonal injury in multiple sclerosis. J Neurol Neurosurg Psychiatry 2003;74:695-697.

5. Bruck W, Bitsch A, Kolenda H, Bruck Y, Stiefel M, Lassmann H. Inflammatory central nervous system demyelina- 
tion: correlation of magnetic resonance imaging findings with lesion pathology. Ann Neurol 1997;42:783-793.

6. van Walderveen MA, Kamphorst W, Scheltens P, et al. Histopathologic correlate of hypointense lesions on T1weighted spin-echo MRI in multiple sclerosis. Neurology 1998;50:1282-1288.

7. Bitsch A, Kuhlmann T, Stadelmann C, Lassmann H, Lucchinetti C, Bruck W. A longitudinal MRI study of histopathologically defined hypointense multiple sclerosis lesions. Ann Neurol 2001;49:793-796.

8. Bagnato F, Jeffries N, Richert ND, et al. Evolution of T1 black holes in patients with multiple sclerosis imaged monthly for 4 years. Brain 2003;126:1782-1789.

9. Cadavid D, Cheriyan J, Skurnick J, Lincoln JA, Wolansky LJ, Cook SD. New acute and chronic black holes in patients with multiple sclerosis randomised to interferon beta-1b or glatiramer acetate. J Neurol Neurosurg Psychiatry 2009;80:1337-1343.

10. Budde MD, Xie M, Cross AH, Song SK. Axial diffusivity is the primary correlate of axonal injury in the experimental autoimmune encephalomyelitis spinal cord: a quantitative pixelwise analysis. J Neurosci 2009;29:2805-2813.

11. Song SK, Sun SW, Ju WK, Lin SJ, Cross AH, Neufeld $\mathrm{AH}$. Diffusion tensor imaging detects and differentiates axon and myelin degeneration in mouse optic nerve after retinal ischemia. Neuroimage 2003;20:1714-1722.

12. Song SK, Sun SW, Ramsbottom MJ, Chang C, Russell J, Cross AH. Dysmyelination revealed through MRI as increased radial (but unchanged axial) diffusion of water. Neuroimage 2002;17:1429-1436.

13. Sun SW, Liang HF, Cross AH, Song SK. Evolving Wallerian degeneration after transient retinal ischemia in mice characterized by diffusion tensor imaging. Neuroimage 2008;40:1-10

14. Zhang JY, Jones M, DeBoy CA, et al. Diffusion tensor magnetic resonance imaging of Wallerian degeneration in rat spinal cord after dorsal root axotomy. J Neurosci 2009; 29:3160-3171.

15. van Waesberghe JH, Kamphorst W, De Groot CJ, et al. Axonal loss in multiple sclerosis lesions: magnetic resonance imaging insights into substrates of disability. Ann Neurol 1999;46:747-754.

16. Bitsch A, Kuhlmann T, Stadelmann C, Lassmann H, Lucchinetti C, Bruck W. A longitudinal MRI study of histopathologically defined hypointense multiple sclerosis lesions. Ann Neurol 2001;49:793-796.

17. van Walderveen MA, Barkhof F, Pouwels PJ, van Schijndel RA, Polman CH, Castelijns JA. Neuronal damage in T1-hypointense multiple sclerosis lesions demonstrated in vivo using proton magnetic resonance spectroscopy. Ann Neurol 1999;46:79-87.

18. Brex PA, Parker GJ, Leary SM, et al. Lesion heterogeneity in multiple sclerosis: a study of the relations between appearances on T1 weighted images, T1 relaxation times, and metabolite concentrations J Neurol Neurosurg Psychiatry 2000;68:627-632.

19. Loevner LA, Grossman RI, McGowan JC, Ramer KN, Cohen JA. Characterization of multiple sclerosis plaques with T1-weighted MR and quantitative magnetization transfer. AJNR Am J Neuroradiol 1995;16:1473-1479.

20. Rovira A, Alonso J, Cucurella G, et al. Evolution of multiple sclerosis lesions on serial contrast-enhanced T1weighted and magnetization-transfer MR images. AJNR Am J Neuroradiol 1999;20:1939-1945.
21. Larsson HB, Thomsen C, Frederiksen J, Stubgaard M, Henriksen $\mathrm{O}$. In vivo magnetic resonance diffusion measurement in the brain of patients with multiple sclerosis. Magn Reson Imaging 1992;10:7-12.

22. Christiansen P, Gideon P, Thomsen C, Stubgaard M, Henriksen $\mathrm{O}$, Larsson $\mathrm{HB}$. Increased water self-diffusion in chronic plaques and in apparently normal white matter in patients with multiple sclerosis. Acta Neurol Scand 1993;87:195-199.

23. Horsfield MA, Larsson HB, Jones DK, Gass A. Diffusion magnetic resonance imaging in multiple sclerosis. J Neurol Neurosurg Psychiatry 1998;64 suppl 1:S80-S84.

24. Tievsky AL, Ptak T, Farkas J. Investigation of apparent diffusion coefficient and diffusion tensor anisotropy in acute and chronic multiple sclerosis lesions. AJNR Am J Neuroradiol 1999;20:1491-1499.

25. Castriota-Scanderbeg A, Fasano F, Hagberg G, Nocentini U, Filippi M, Caltagirone C. Coefficient $\mathrm{D}(\mathrm{av})$ is more sensitive than fractional anisotropy in monitoring progression of irreversible tissue damage in focal nonactive multiple sclerosis lesions. AJNR Am J Neuroradiol 2003;24:663-670.

26. Filippi M, Iannucci G, Cercignani M, Assunta Rocca M, Pratesi A, Comi G. A quantitative study of water diffusion in multiple sclerosis lesions and normal-appearing white matter using echo-planar imaging. Arch Neurol 2000;57: 1017-1021.

27. Droogan AG, Clark CA, Werring DJ, Barker GJ, McDonald WI, Miller DH. Comparison of multiple sclerosis clinical subgroups using navigated spin echo diffusion-weighted imaging. Magn Reson Imaging 1999;17:653-661.

28. Nusbaum AO, Lu D, Tang CY, Atlas SW. Quantitative diffusion measurements in focal multiple sclerosis lesions: correlations with appearance on TI-weighted MR images. AJR Am J Roentgenol 2000;175:821-825.

29. Castriota Scanderbeg A, Tomaiuolo F, Sabatini U, Nocentini U, Grasso MG, Caltagirone C. Demyelinating plaques in relapsing-remitting and secondary-progressive multiple sclerosis: assessment with diffusion MR imaging. AJNR Am J Neuroradiol 2000;21:862-868.

30. Truyen $L$, van Waesberghe JH, van Walderveen MA, et al. Accumulation of hypointense lesions ("black holes") on T1 spin-echo MRI correlates with disease progression in multiple sclerosis. Neurology 1996;47:1469-1476.

31. van Walderveen MA, Barkhof F, Hommes OR, et al. Correlating MRI and clinical disease activity in multiple sclerosis: relevance of hypointense lesions on short-TR/ short-TE (T1-weighted) spin-echo images. Neurology 1995;45:1684-1690.

32. Iannucci G, Minicucci L, Rodegher M, Sormani MP, Comi G, Filippi M. Correlations between clinical and MRI involvement in multiple sclerosis: assessment using T(1), T(2) and MT histograms. J Neurol Sci 1999;171: 121-129.

33. Naismith RT, Cross AH. Multiple sclerosis and black holes: connecting the pixels. Arch Neurol 2005;62:16661668 .

34. Naismith RT, Xu J, Tutlam NT, et al. Disability in optic neuritis correlates with diffusion tensor-derived directional diffusivities. Neurology 2009;72:589-594.

35. Naismith RT, Xu J, Tutlam NT, Trinkaus K, Cross AH, Song S-K. Radial diffusivity in remote optic neuritis discriminates visual outcomes. Neurology 2010;74:1702-1710.

36. Werring DJ, Brassat D, Droogan AG, et al. The pathogenesis of lesions and normal-appearing white matter changes 
in multiple sclerosis: a serial diffusion MRI study. Brain 2000;123:1667-1676.

37. Wheeler-Kingshott CA, Cercignani M. About "axial" and "radial" diffusivities. Magn Reson Med 2009;61:12551260.

38. Dousset V, Gayou A, Brochet B, Caille JM. Early structural changes in acute MS lesions assessed by serial magnetization transfer studies. Neurology 1998;51:1150-1155.
39. Silver NC, Lai M, Symms MR, Barker GJ, McDonald WI, Miller DH. Serial magnetization transfer imaging to characterize the early evolution of new MS lesions. Neurology 1998;51:758-764.

40. Richert ND, Ostuni JL, Bash CN, Leist TP, McFarland HF, Frank JA. Interferon beta- $1 \mathrm{~b}$ and intravenous methylprednisolone promote lesion recovery in multiple sclerosis. Mult Scler 2001;7:49-58.

\section{Visit the Newly Designed Neurology ${ }^{\circledR}$ Web Site at www.neurology.org}

- Enhanced navigation format

- Increased search capability

- Highlighted articles

- Detailed podcast descriptions

- RSS Feeds of current issue and podcasts

- Mobile device download link

- AAN Web page links

- Links to Neurology Now ${ }^{\circledR}$, Neurology Today ${ }^{\circledR}$, and Continuum ${ }^{\circledR}$

- Resident \& Fellow subsite

Social media for Neurology ${ }^{\circledR}$ has been initiated with the launch of the Web site:

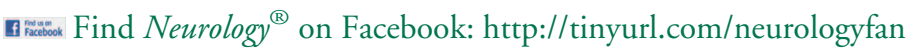

twitter Follow Neurology ${ }^{\circledR}$ on Twitter: http://twitter.com/GreenJournal 